\title{
Investigation of branching structure formation by solutions of a mathematical model of pattern formation in coral reefs
}

\author{
L.W. Somathilake ${ }^{1^{*}}$ and J.R. Wedagedera ${ }^{2}$ \\ ${ }^{I}$ Department of Mathematics, Faculty of Science, University of Ruhuna, Matara. \\ ${ }^{2}$ Simcyp-CERTARA Limited, Blades Enterprise Centre, John Street, Sheffield S2 4SU, United Kingdom.
}

Revised: 18 January 2016; Accepted: 19 May 2016

\begin{abstract}
A reaction-diffusion type mathematical model for the growth of corals in a tank, describing the spatial time evolution of the biomass of dissolved nutrients (food of polyps) and dissolved solid materials (calcium carbonate) of the tank, is considered. Some properties of the spatial patterns when the model parameters lie in the Turing space are investigated based on dispersion relation and unstable wave numbers of the linearised system. Branching structure formation process in the model is explained analytically. The model is solved numerically in one dimension subject to no-flux boundary conditions and it is shown that the numerical results agree with the analytically derived properties of the solutions.
\end{abstract}

Keywords: Dispersion relation, reaction-diffusion equations, spatial temporal pattern formation, Turing instability.

\section{INTRODUCTION}

Stony corals are built by colonies of several thousands of interconnected individuals known as polyps. Colonies develop when a planktonic coral larva, called a planula, settles on a hard surface. All coral polyps have the same basic body structure including tentacles with stingy cells that surround a mouth at the top of the central body. Polyps of hard corals live in a rigid skeleton called calices, made up of calcium carbonate. The rigid skeleton is formed when the dissolved calcium carbonate ions in the water are extracted and deposited by the polyps. Polyps live on the surface and within the top few centimeters of the skeleton. As new polyps are formed, they build new calices to reside on top of the old skeleton and this process cause the growth of coral (Merks et al., 2004). The skeletal structures of the corals or the morphogenesis of corals is very complex. This complexity makes it harder to model the process of coral morphology. However, various modelling approaches on coral morphogenesis processes have been previously reported (Kaandorp et al., 1996; 2005; 2008; Mistr \& Bercovici, 2003; Merks, 2003a; b; c; 2010; Chindapol et al., 2013). A reaction diffusion type mathematical model for the growth of corals in a tank has been previously proposed by Somathilake and Wedagedera $(2012 ; 2014)$ considering the nutrient polyps interaction. This model has been derived based on the model proposed by Mistr and Bercovici (2003). The nondimensionalised form of this mathematical model takes the form

$$
\left.\begin{array}{l}
\frac{\partial u}{\partial t}=\Delta u+1-u-\alpha^{2} v^{2} u, \quad x \in \Omega \subset \mathbb{R}^{2}, \quad t>0 \\
\frac{\partial v}{\partial t}=d \Delta v-\lambda v+\alpha^{2} v^{2} u, \quad x \in \Omega \subset \mathbb{R}^{2}, \quad t>0 \\
\frac{\partial w}{\partial t}=\lambda_{1} v, \quad x \in \Omega \subset \mathbb{R}^{2}, \quad t>0 .
\end{array}\right\}
$$

Here, $\Omega$ is the model geometry, $u, v$ and $w$ are vertically averaged nondimensionalised concentrations of dissolved nutrients (foods of coral polyps), dissolved calcium carbonate ions, and the biomass of calcium carbonate deposited by coral polyps, respectively. The vertical averaging process was done by assuming specific distribution functions. $\Delta=\frac{\partial^{2}}{\partial x^{2}}+\frac{\partial^{2}}{\partial y^{2}}$ is the Laplacian operator and the parameters are defined as $d=\frac{D_{v}}{D_{u}}, \alpha^{2}=\frac{k H^{2} u_{s}^{2}}{3 D_{u}}, \lambda=d+\frac{k_{1} H^{2}}{3 D_{u}}, \lambda_{1}=\frac{k_{1} H^{2}}{3 D_{u}}$. Here $H$ is the height of the tank, $D_{v}$ and $D_{u}$ are diffusion rates of dissolved nutrients and calcium carbonate ions, respectively, and $k, k_{1}$ are positive constants related to reaction rates of the components (Somathilake \&

*Corresponding author (sthilake@maths.ruh.ac.lk) 
Wedagedera, 2014). If the spatial diffusion process of the components is ignored (assuming homogeneous medium or well stirred medium) then system (1) is reduced to a system of ordinary differential equations of the form:

$$
\left.\begin{array}{l}
\frac{d u}{d t}=1-u-\alpha^{2} u v^{2} \\
\frac{d v}{d t}=-\lambda v+\alpha^{2} u v^{2} \\
\frac{d w}{d t}=\lambda_{1} v .
\end{array}\right\}
$$

The local and global stability of the solutions of the system of ordinary differential equations (2) have been discussed in a previous study (Somathilake \& Wedagedera, 2012). Instability analysis and pattern formation behaviour in the Turing sense of the model (1), subject to the following boundary conditions have been discussed previously (Somathilake \& Wedagedera, 2014).

$$
\left.\begin{array}{ll}
\nabla u \cdot \mathbf{n}=0, & x \in \partial \Omega, \\
\nabla v \cdot \mathbf{n}=0, & x \in \partial \Omega,
\end{array}\right\}
$$

Here $\nabla$ denotes the gradient operator and $\mathbf{n}$ denotes the outward unit normal vector to the domain boundary $\partial \Omega$.

In this paper, some properties of the spatial temporal patterns generated by the proposed model when the parameters lie in the Turing space are discussed based on the dispersion relation and unstable wave numbers of the linearised system. A brief introduction on dispersion relation and turing instability conditions (Turing, 1952; Murray, 2003) is given later. Critical values of $d$ are derived when the parameters lie in the Turing space of the model. The possible range of unstable wave numbers of the wave-like solutions of the linearised system about the steady state $\mathrm{S}_{2}$ (see next section) are derived and the possible form of analytical solution for the perturbed system is presented. A brief introduction on the numerical tool used in this paper is given. The variation of critical values of $d$ are explained and the variation of the wave numbers of the wave type solutions, with respect to the model parameters are explained. The variation of the growth rates of the solutions of the model are explained based on the linearised system about a steady state. The numerical solutions of the proposed model are also presented.

\section{METHODOLOGY}

The model considered is a reaction diffusion system (RDS). The behaviour of the solutions of reaction diffusion systems (RDSs) can be predicted using the perturbed solutions of the system about steady states. The properties of perturbed systems can be explained via Turing instability conditions (Turing, 1952; Murray, 2003 ) and dispersion relation of the model. The solutions of RDSs form spatial time patterns if the parameters of the model satisfy the Turing instability conditions. Turing instability conditions and dispersion relation of the model are presented below:

\section{Turing instability and dispersion relation}

In system (1) the term $w$ in the third equation depends only on time and $v$. If $v$ is known, $w$ can be obtained by the time integration of $v$. Therefore, it is sufficient to consider the first two equations in the analysis. Rewrite the first two equations of the system (1) in the form:

$$
\left.\begin{array}{ll}
\frac{\partial u}{\partial t}=\Delta u+f(u, v), & x \in \Omega \subset \mathbb{R}^{2}, \quad t>0 \\
\frac{\partial v}{\partial t}=d \Delta v+g(u, v), & x \in \Omega \subset \mathbb{R}^{2}, \quad t>0
\end{array}\right\} .
$$

where $f(u, v)=1-u-\alpha^{2} v^{2} u$ and $g(u, v)=-\lambda v+\alpha^{2} v^{2} u$.

There are three steady states: $S_{1} \equiv\left(u_{s 1}, v_{s 1}\right), S_{2} \equiv\left(u_{s 2}, v_{s 2}\right)$ and $S_{3} \equiv\left(u_{s 3}, v_{s 3}\right)$ for the spatially homogeneous case. Here $u_{s 1}=1, v_{s 1}=0$, and for $\alpha>2 \lambda, \quad u_{s 2}=\frac{\alpha-\sqrt{\alpha^{2}-4 \lambda^{2}}}{2 \alpha}, \quad v_{s 2}=\frac{\alpha+\sqrt{\alpha^{2}-4 \lambda^{2}}}{2 \alpha \lambda}$, $u_{s 3}=\frac{\alpha+\sqrt{\alpha^{2}-4 \lambda^{2}}}{2 \alpha}$ and $v_{s 3}=\frac{\alpha-\sqrt{\alpha^{2}-4 \lambda^{2}}}{2 \alpha \lambda}$.

It can be shown that the trivial steady state $S_{1}$ is a stable node and when $\alpha>2 \lambda, S_{3}$ is a saddle point. The stability of $S_{2}$ depends on the parameters $\alpha$ and $\lambda$ (Somathilake \& Wedagedera, 2012). Therefore, Turing type instability may occur only at $S_{2}$. Considering the perturbation $u=u_{s 2}+u_{1}, v=v_{s 2}+v_{1}$ at $S_{2}$, systems (4) and (3) can be linearised in the form:

$$
\begin{gathered}
\frac{\partial \mathbf{w}}{\partial t}=D \Delta \mathbf{w}+A \mathbf{w} \quad x \in \Omega, t>0, \\
\nabla \mathbf{w}=0, \quad x \in \partial \Omega, \quad t>0, \quad i=1,2,
\end{gathered}
$$

where, $\mathbf{w}=\left(u_{1}, v_{1}\right)^{T}$ and

$$
A=\left(\begin{array}{ll}
a_{11} & a_{12} \\
a_{21} & a_{22}
\end{array}\right)=\left.\left(\begin{array}{ll}
\frac{\partial f_{1}}{\partial u} & \frac{\partial f_{1}}{\partial v} \\
\frac{\partial \partial f_{2}}{\partial u} & \frac{\partial f_{2}}{\partial v}
\end{array}\right)\right|_{S_{2}} .
$$

We assume that solutions of the perturbed system (5) take the form:

$$
\left(u_{1}, v_{1}\right)=(A, B) e^{i \mathbf{k} \cdot \mathbf{r}+\sigma t},
$$


where $\sigma$ is the growth rate, $\mathbf{k}=\left(k_{1}, k_{2}\right)$ is the wave number vector and $\mathbf{r}=(x, y)$ is the position vector. Substituting equation (8) in system (5) we get the dispersion relation (Engelhardt, 1994; Murray, 2003) in the form:

$$
\sigma^{2}+A_{1}(\mu) \sigma+h(\mu)=0
$$

where $\mu=k^{2}=k_{1}^{2}+k_{2}^{2}$,

$$
\begin{aligned}
A_{1}(\mu) & =\frac{\left(\alpha^{2}-2 \lambda^{3}+\alpha \sqrt{\alpha^{2}-4 \lambda^{2}}+2 \lambda^{2} \mu+2 d \lambda^{2} \mu\right)}{2 \lambda^{2}} \\
h(\mu) & =\frac{\alpha^{2}(\lambda+d \mu)+\alpha \sqrt{\alpha^{2}-4 \lambda^{2}}(\lambda+d \mu)-2 \lambda^{2}\left(-d \mu^{2}+\lambda(2+\mu)\right)}{2 \lambda^{2}} .
\end{aligned}
$$

Also, Turing instability conditions of the system (4) at $\mathrm{S}_{2}$ can be derived in the form:

$$
\begin{aligned}
& T C_{1} \equiv \frac{\alpha^{2}-4 \lambda^{2}+\alpha \sqrt{\alpha^{2}-4 \lambda^{2}}}{2 \lambda}>0, \\
& T C_{2} \equiv-\frac{\alpha^{2}-2 \lambda^{3}+\alpha \sqrt{\alpha^{2}-4 \lambda^{2}}}{2 \lambda^{2}}<0, \\
& T C_{3} \equiv \frac{2 \lambda^{3}-\alpha d\left(\alpha+\sqrt{\alpha^{2}-4 \lambda^{2}}\right)}{2 \lambda^{2}}>0, \\
& T C_{4} \equiv\left(\frac{2 \lambda^{3}-d \alpha\left(\alpha+\sqrt{\alpha^{2}-4 \lambda^{2}}\right)}{2 \lambda^{2}}\right)^{2}-4 d\left(\frac{\alpha^{2}-4 \lambda^{2}+\alpha \sqrt{\alpha^{2}-4 \lambda^{2}}}{2 \lambda}\right)>0 .
\end{aligned}
$$

Turing space (the parameter space in which the Turing instability conditions are satisfied) can be written as the union of two regions $\mathcal{R}_{1}$ and $\mathcal{R}_{2}$ defined as:

$\mathcal{R}_{1}$ : The area within the line $\alpha=2 \lambda$ and the curve $\alpha=\alpha_{2}$ for $\lambda \leq 2$. That is

$\mathcal{R}_{1}=\left\{(\lambda, \alpha) ; \quad 2 d \leq \lambda \leq 2, \quad 2 \lambda<\alpha<\alpha_{2}\right\}$.

$\mathcal{R}_{2}$ : The area covered by the curves $\alpha=\alpha_{1}$ and $\alpha=\alpha_{2}$ for $\lambda>2$. That is

$\mathcal{R}_{2}=\left\{(\lambda, \alpha) ; \quad \lambda>2, \quad \alpha_{1}<\alpha<\alpha_{2}\right\}$

where $\alpha_{1}=\frac{\lambda^{2}}{\sqrt{\lambda-1}}$ and

$\alpha_{2}=\frac{\sqrt{d \lambda^{3}\left(8 d^{2}+7 d \lambda+3 \lambda^{2}\right)-2 \sqrt{2} d \sqrt{\lambda^{7}(\lambda-d)(2 d+\lambda)^{2}}}}{d(d+\lambda)}$

are two positive solutions for $\alpha$ of the equations of $T C_{2}=$ 0 and $T C_{4}=0$, respectively (Somathilake \& Wedagedera, 2014).

\section{Critical values for $d$}

The condition $T C_{3}$ gives us $d<d_{1}$, where $d_{1}=\frac{2 \lambda^{3}}{\alpha\left(\alpha+\sqrt{\alpha^{2}-4 \lambda^{2}}\right)}$. Also the condition $T C_{4}$ implies $d<d_{2}$ or $d>d_{3}$.

Here $\quad d_{2}=\frac{3 \alpha^{2} \lambda^{3}+3 \alpha \lambda^{3} \sqrt{\alpha^{2}-4 \lambda^{2}}-\mathcal{A}}{\alpha^{2}\left(\alpha^{2}-2 \lambda^{2}+\alpha \sqrt{\alpha^{2}-4 \lambda^{2}}\right)}$ and

$$
d_{3}=\frac{3 \alpha^{2} \lambda^{3}+3 \alpha \lambda^{3} \sqrt{\alpha^{2}-4 \lambda^{2}}+\mathcal{A}}{\alpha^{2}\left(\alpha^{2}-2 \lambda^{2}+\alpha \sqrt{\alpha^{2}-4 \lambda^{2}}\right)}, \text { where }
$$

$\mathcal{A}=4\left(2 \lambda^{5}+\sqrt{\lambda^{6}\left(\alpha^{4}-5 \alpha^{2} \lambda^{2}+4 \lambda^{4}+\alpha^{3} \sqrt{\alpha^{2}-4 \lambda^{2}}-3 \alpha \lambda^{2} \sqrt{\alpha^{2}-4 \lambda^{2}}\right)}\right)$.

It can be shown that $d_{2}<d_{1}$. Therefore, in order to satisfy both conditions $T C_{3}$ and $T C_{4}$ it is needed that $d<d_{2} \equiv d_{c}(\alpha, \lambda)$ (say).

\section{Range of unstable wave numbers}

The $h(\mu)$ curves against $\mu$ at different levels of $d$ when $\lambda=2.1, \alpha=4.24$, are shown in Figure 1. It can be shown that $d_{c}(4.20477,2.1)=0.648532$.

For $d<d_{c}$ we get two roots $\mu_{1}$ and $\mu_{2}$ for $h(\mu)=0$, and no real roots for $\mu$ when $d>d_{c}$

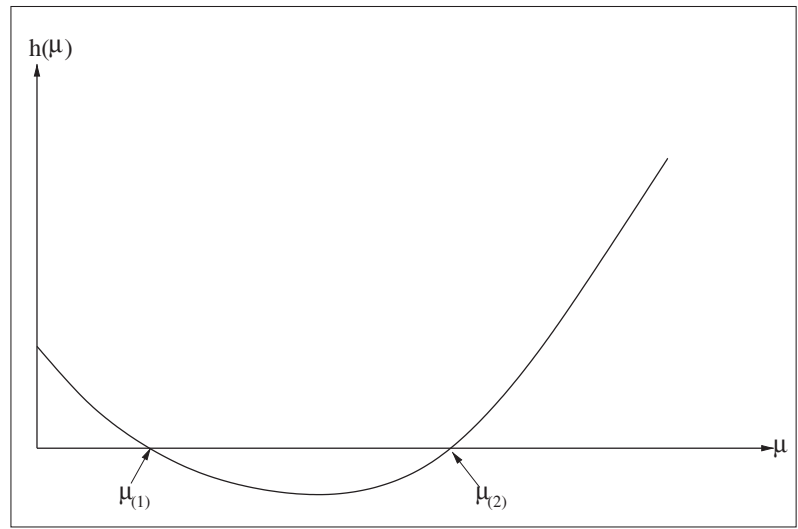

(a)

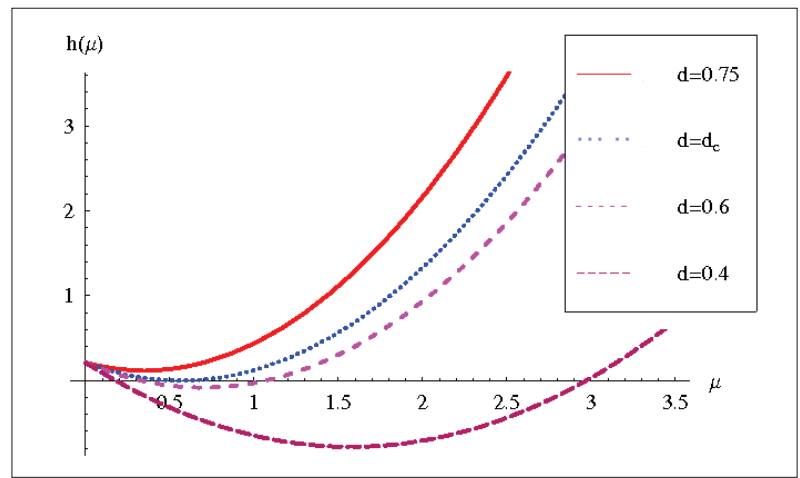

(b)

Figure 1: (a) Sketch of the sensitivity of $h(\mu)$ with respect to $\mu$; (b) $h(\mu)$ curves from top to bottom are for $d=0.75>d_{c}, d$ $=d_{c}=0.648532, d=0.6<d_{c}$ and $d=0.4<d_{c}$, respectively. 
where,

$$
\begin{aligned}
& \mu_{(1)}=\frac{-\left(d \alpha^{2}\right)+2 \lambda^{3}-d \alpha \sqrt{\alpha^{2}-4 \lambda^{2}}+A}{4 d \lambda^{2}}, \\
& \mu_{(2)}=\frac{-\left(d \alpha^{2}-2 \lambda^{3}+d \alpha \sqrt{\alpha^{2}-4 \lambda^{2}}+A\right)}{4 d \lambda^{2}} .
\end{aligned}
$$

Here

$$
A=\sqrt{-8 d \lambda^{3}\left(\alpha^{2}-4 \lambda^{2}+\alpha \sqrt{\alpha^{2}-4 \lambda^{2}}\right)+\left(-2 \lambda^{3}+d \alpha\left(\alpha+\sqrt{\alpha^{2}-4 \lambda^{2}}\right)\right)^{2}} .
$$

It can be observed from Figure 1 that $\mu_{2}$ increases and $\mu_{1}$ decreases as $d$ decreases. That is the range where unstable wave numbers increases as $d$ decreases. Solving equation (9) for $\sigma$ we get two solutions $\sigma_{1}$ and $\sigma_{2}$ given by

$$
\begin{gathered}
\sigma_{1}=\frac{-A_{1}+\sqrt{A_{1}^{2}-4 h(\mu)}}{2}, \\
\sigma_{2}=\frac{-A_{1}-\sqrt{A_{1}^{2}-4 h(\mu)}}{2} .
\end{gathered}
$$

It is clear that $\sigma_{1}>0$ and $\sigma_{2}<0$ when $h(u)<0$ (i.e. $\left.\mu_{(1)}<\mu<\mu_{(2)}\right)$. Since $\sigma_{1}>0$, the solution of the system is unstable.

\section{Solutions of the perturbed system}

Now we consider the system (4) subject to boundary conditions (3) in one dimensional domain $\Omega=(0, L)$. Then the eigenvalues and eigenfunction for the Laplacian on $\Omega$ under noflux (zero Neumann) boundary conditions are: $\mu_{j}=\left(\frac{j \pi}{L}\right)^{2}$ and $\phi_{j}=\cos \left(\frac{j \pi x}{L}\right), j=0,1,2, \ldots$ respectively. Then, the solutions of (5) subject to the boundary conditions (6) can be written in the form:

$$
\left.\begin{array}{l}
u_{1}(x, t) \simeq \sum_{j=0}^{\infty} a_{j} e^{\sigma\left(\mu_{j}\right) t} \cos \left(\sqrt{\mu_{j}} x\right) \\
v_{1}(x, t) \simeq \sum_{j=0}^{\infty} b_{j} e^{\sigma\left(\mu_{j}\right) t} \cos \left(\sqrt{\mu_{j}} x\right)
\end{array}\right\} .
$$

Here $\sigma$ is the solution of equation (9) and $a_{j}$ and $b_{j}$ are constants, which are determined by considering the Fourier series of $u_{1}, v_{1}$ and initial conditions.

The $j^{\text {th }}$ mode solution of equation (5) subject to the zero flux boundary condition (equation 6) exists when $L$ satisfy $\mu_{(1)}<\mu_{j}=\left(\frac{j \pi}{L}\right)^{2}<\mu_{(2)}$. For given $L$, let $n_{1}$ be the smallest integer, $j$, such that $\frac{L \sqrt{\mu_{(1)}}}{\pi}<j$ and $n_{2}$ be the largest integer, $j$, such that $j<\frac{L \sqrt{\mu_{(2)}}}{\pi}$. Then spatially heterogeneous solutions of the system takes the form:

$$
\left.\begin{array}{l}
u_{1}(x, t) \simeq \sum_{\substack{n=n_{1} \\
n=n_{2}}}^{n=n_{2}} a_{n} e^{\sigma\left(\mu_{n}\right) t} \cos \left(\sqrt{\mu_{n}} x\right) \\
v_{1}(x, t) \simeq \sum_{n=n_{1}} b_{n} e^{\sigma\left(\mu_{n}\right) t} \cos \left(\sqrt{\mu_{n}} x\right)
\end{array}\right\} .
$$

The possible smallest wave number is $\frac{\pi}{L}$; that correspond to $j=1$. If $L$ is sufficiently small such that $L<\frac{\pi}{\sqrt{\mu_{(2)}}}$ then for given $\alpha, \lambda$ and $d$ there does not exist any spatially heterogeneous solution. That is $u(x, t)$ and $v(x, t)$ tend to corresponding steady state exponentially. Therefore, for given parameters $\alpha, \lambda$ and $d$ the critical domain size is $L_{c}=\frac{\pi}{\sqrt{\mu_{(2)}}}$.

\section{Numerical simulation}

System (4) subject to boundary conditions (3) is numerically solved in one dimensional space using MATLAB built in function, pdepe. Errors can be controlled within pdepe by adjusting the absolute error and relative error in each step of the numerical iteration. Relative and absolute errors are defined as RelErr $=\frac{\left|x_{i+1}-x_{i}\right|}{\left|x_{i}\right|}$ and AbsErr $=\left|x_{i+1}-x_{i}\right|$, where $x_{i}$ is the computed value of a dependent variable $x$ at the $i^{\text {th }}$ step of a numerical iteration process. Absolute error and relative error are controlled by introducing their respective tolerances AbsTol and RelTol. The condition $\frac{\left|x_{i+1}-x_{i}\right|}{\left|x_{i}\right|}<$ RelTol checks what fraction of the new solution has changed since the last iteration. But if the solution is around zero then the relative error becomes problematic since $\frac{x}{0}$ is undefined. In such a case absolute error is useful in controlling errors.

In the numerical iteration process space and time are discretised with step sizes $\Delta x$ and $\Delta t$, respectively. A small perturbation from $S_{2}$ of the form

$$
\begin{aligned}
& u(x, 0)=u_{02} \quad x \in \Omega, \\
& v(x, 0)=\left\{\begin{array}{ll}
v_{02}+v_{02} \frac{\operatorname{Rand}[0,1]}{100}, & x \in \omega \\
v_{02}, & x \in \Omega \backslash \omega .
\end{array}\right\}
\end{aligned}
$$

is used as initial conditions. Here $\omega$ is an open subset of $\Omega$ and Rand $[0,1]$ means a random number between 0 and 1. In our problem $x_{i}=u_{j, i}$ or $x_{i}=v_{j, i}$ over $j$. Where $u_{j, i}$ and $v_{j, i}$ represents the numerical values of $u$ and $v$ at the position $j \Delta x$ and time $i \Delta t$, respectively. 


\section{RESULTS AND DISCUSSION}

\section{The range of the critical value $d_{c}$ of $d$}

Sensitivity of $d_{c}$ with respect to $\alpha$ for different values of $\lambda$ is shown in Figure 2.

Denote

$\alpha_{\text {min }}(\lambda, d)= \begin{cases}2 \lambda, & \text { if } 2 d<\lambda<2 \\ \alpha_{1}(\lambda, d), & \text { if } \lambda \geq 2,\end{cases}$

and

$\alpha_{\text {max }}(\lambda, d)= \begin{cases}2, & \text { if } 2 d<\lambda<2 \\ \alpha_{2}(\lambda, d), & \text { if } \lambda \geq 2 .\end{cases}$

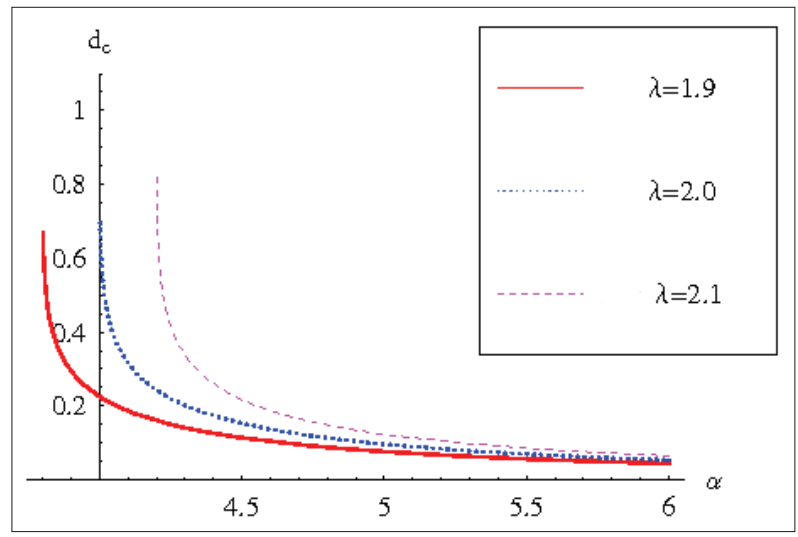

Figure 2: The sensitivity of $d_{c}$ with respect to $\alpha$ at different levels of $\lambda$.
That is $\left(\alpha_{\min }(\lambda, d), \alpha_{\max }(\lambda, d)\right)$ is the range of $\alpha$ in the Turing space for given $\lambda$ and $d$. For given $\lambda$ the value of $d_{c}$ is maximised at $\alpha=\alpha_{\min }(\lambda)$. For given $\lambda$, the maximum of $d_{c}$ denote as $\max \left(d_{c}\right)(\lambda)$. That is

$$
\max \left(d_{c}\right)(\lambda)=d_{c}\left(\alpha_{\min }(\lambda), \lambda\right)
$$

The $\max \left(d_{c}\right)(\lambda)$ is maximised at $\lambda=2$. The value of $\max \left(d_{c}\right)(\lambda)$ decreases as $\lambda$ deviates from 2. Also, for fixed $\lambda, d_{c}$ decreases as $\alpha$ increases from $\alpha_{\text {min }}$. That is the possible range for $d$, is $\left(0, d_{c}\right)$ for fixed $\lambda$. Furthermore $d_{c}$ decreases as $\alpha$ increases from $\alpha_{\text {min }}$.

The plot of $\max \left(d_{c}\right)$ against $\lambda$ is shown in Figure 3 . As $\lambda$ increases $\max \left(d_{c}\right)$ tends to $d_{c 1}=3-2 \sqrt{2}$. Also,

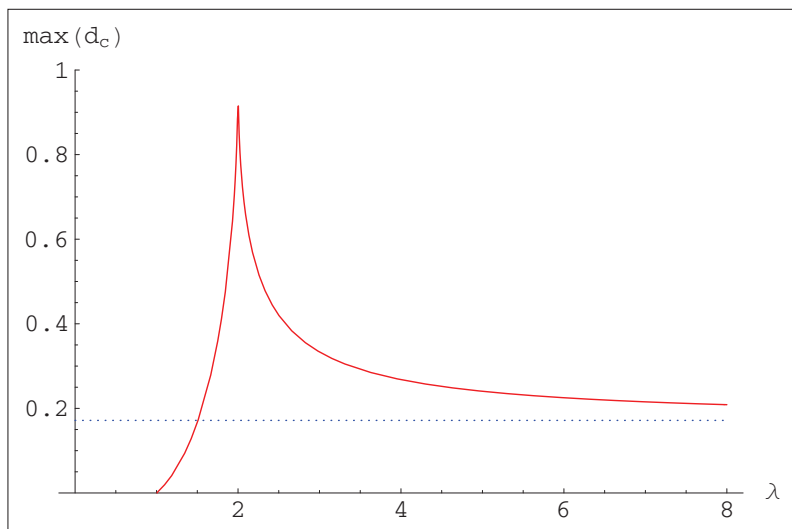

Figure 3: The variation of $\max \left(d_{c}\right)$ with respect to $\lambda$ The dotted horizontal line is $\max \left(d_{c}\right)=(3-2 \sqrt{2})$ line.

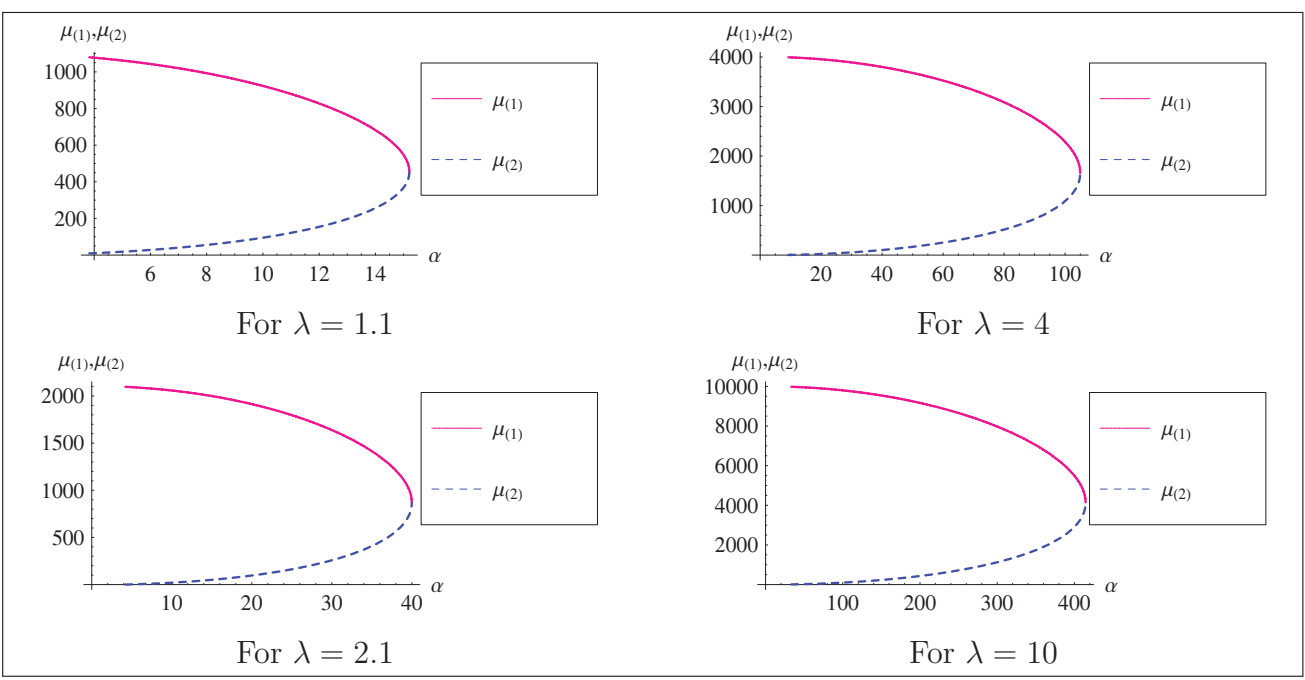

Figure 4: Variation of $\mu_{(1)}$ and $\mu_{(2)}$ with respect to $\alpha$ for different levels of $\lambda$ when $d=0.001$ 
as $\lambda$ tends to 1 the $\max \left(d_{c}\right)$ tends to 0 (zero) and as $\lambda$ tends to 2 the $\max \left(d_{c}\right)$ tends to 1 . That is, in order to exist spatial patterns $d=\frac{D_{v}}{D_{u}}$ should be less than 1 . In other words, diffusion rate of the dissolved calcium carbonate should be less than that of dissolved nutrients. This condition is acceptable with physical situations.

\section{Variation of wave numbers}

The $\mu_{(1)}$ and $\mu_{(2)}$ curves with respect to $\alpha$ for different values of $\lambda$ when $d=0.001$ are shown in Figure 4 . It can be observed that for the given $\lambda$, the maximum range for $\mu$ is given at $\alpha=\alpha_{\text {min }}$. The possible range of $\mu$ decreases as $\alpha$ increases and vanishes at $\alpha_{\max }$. That is the maximum range of wave numbers for a particular domain for given $\lambda$ and $d$ is given at $\alpha=\alpha_{\min }$. That is, the highest spatial heterogeneity of the solution of system (1) can be expected at $\alpha=\alpha_{\text {min }}$. Also, it can be observed that as $\lambda$ increases the range of $\mu$ and $\alpha$ increases at a fixed value $d<d_{c}$. That is for fixed $d\left(d<d_{c}\right)$ the heterogeneity of the spatial patterns formed by the solutions of system (1) increases as $\lambda$ increases.

The $\mu_{(1)}$ and $\mu_{(2)}$ curves with respect to $\alpha$ for different values of $d$ when $\lambda=2.1$ are shown in Figure 5. According to the figure it can be observed that as $d$ increases the possible ranges of $\alpha$ and $\mu$ decrease. Therefore, for fixed $\lambda$, the heterogeneity of the spatial patterns formed by solutions of system (1) in a particular domain decreases as $d$ increases from 0 to $d_{c}$.

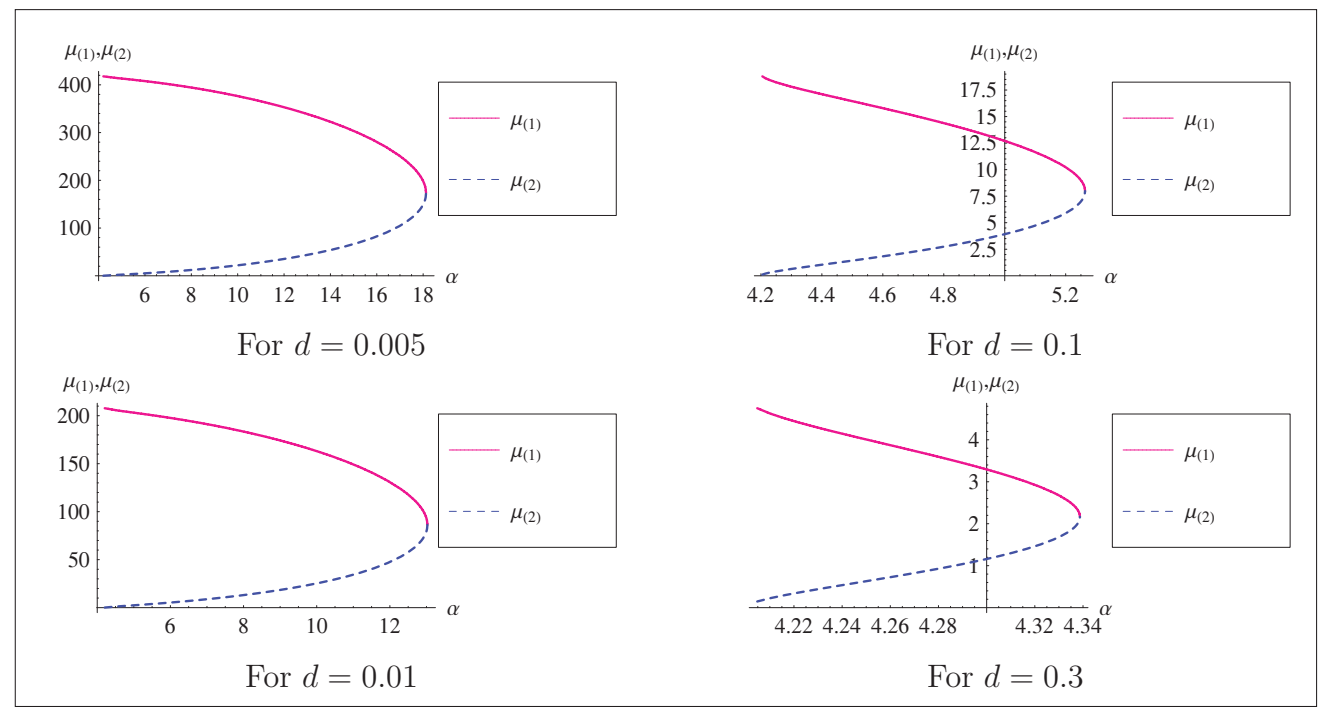

Figure 5: Variation of $\mu_{(1)}$ and $\mu_{(2)}$ with respect to $\alpha$ for different levels of $d$ when $\lambda=2.1$

\section{Maximum growth rates}

Figure 6 shows the plot of real part of $\sigma_{1}, \operatorname{Re}\left(\sigma_{1}\right)$, with respect to $\mu$ at different levels of $\alpha$ when $\lambda=3$. It can be seen that $\operatorname{Re}\left(\sigma_{1}\right)$ is maximised at a particular value of $\mu$ for given $\alpha$ and $\lambda$.

The critical value of $\sigma_{1}$ is occurred when

$$
\frac{d \sigma_{1}}{d \mu}=0
$$

Solving equation (17) for $\mu$ we get two solutions $\mu^{(1)}$ and $\mu^{(2)}$ given by:

$$
\begin{aligned}
\mu^{(1)} & =\frac{A_{2}+B_{2}}{2 d(d-1)^{2} \lambda^{4}}, \\
\mu^{(2)} & =\frac{A_{2}-B_{2}}{2 d(d-1)^{2} \lambda^{4}} .
\end{aligned}
$$

Where

$A_{2}=-d \lambda^{2}\left(\alpha^{2}+2 \lambda^{3}+\alpha \sqrt{\alpha^{2}-4 \lambda^{2}}\right)+d^{2} \lambda^{2}\left(\alpha^{2}+2 \lambda^{3}+\alpha \sqrt{\alpha^{2}-4 \lambda^{2}}\right)$,

$B_{2}=2 \sqrt{d\left(-1+d^{2}\right)^{2} \lambda^{7}\left(\alpha^{2}-2 \lambda^{2}+\alpha \sqrt{\alpha^{2}-4 \lambda^{2}}\right)}$. 


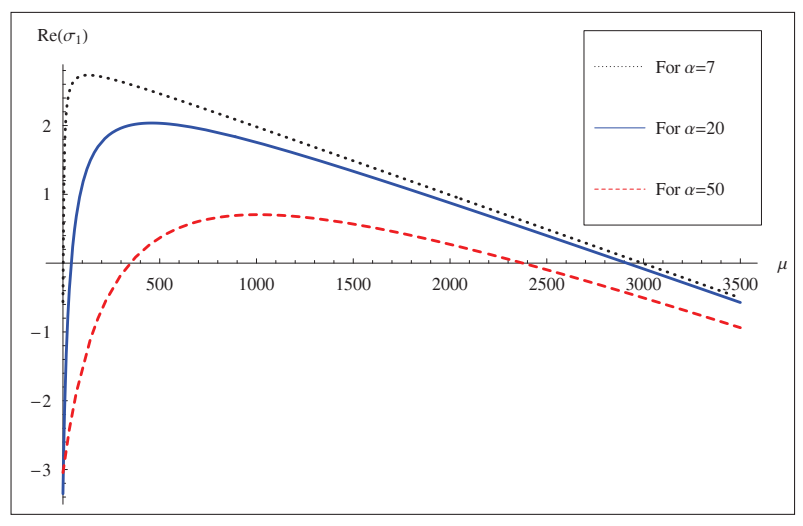

Figure 6: The variation of $\operatorname{Re}\left(\sigma_{1}\right)$ against $\mu$ at different levels of $\alpha$

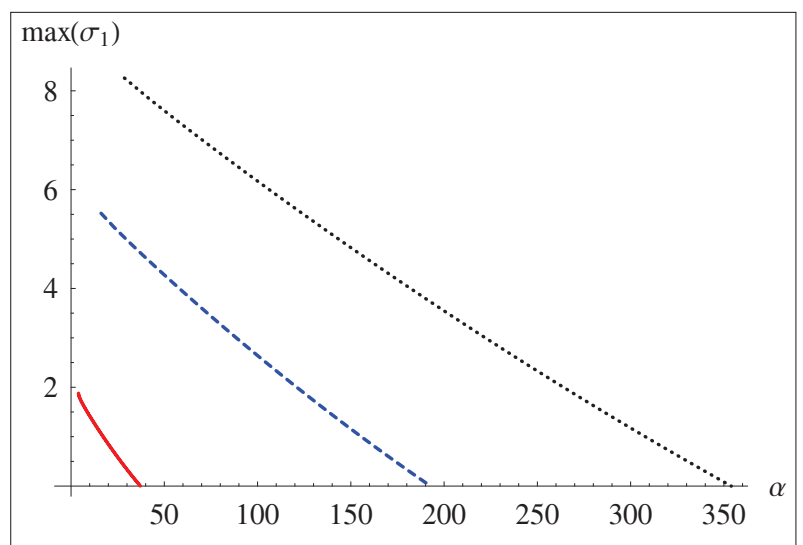

Figure 7: The variation of $\max \left(\sigma_{1}\right)$ against $\alpha$ at different levels of $\lambda$ : The curves from bottom to top are for the levels $\lambda=2$, $\lambda=6$ and $\lambda=9$, respectively.

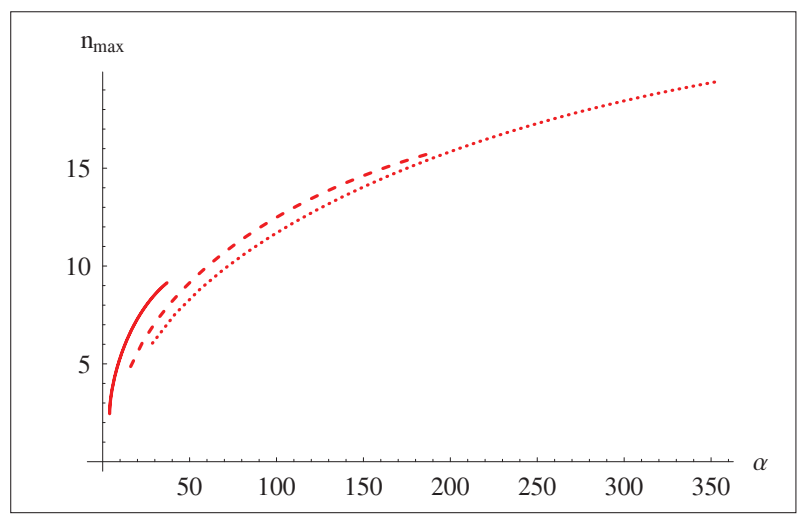

Figure 8: The variation of $n_{\max }$ with respect to different levels of $\lambda$ : The curves from left to right are for $\lambda=2, \lambda=6, \lambda=9$, respectively.
It can be shown that $\sigma_{1}$ is maximised at $\mu=\mu^{(1)}$. That is $\max \left(\sigma_{1}\right)=\left.\sigma_{1}\right|_{\mu=\mu^{(1)}}$. The plots of $\max \left(\sigma_{1}\right)$ against $\alpha$ for different values of $\lambda$ are shown in Figure 7.

It is clear that the growth rate is maximised at $\sigma_{1}=\max \left(\sigma_{1}\right)$. According to Figure 7, for a given $\lambda$, $\max \left(\sigma_{1}\right)$ is maximised at $\alpha_{\min }(\lambda)$, the possible minimum value of $\alpha$. The wavelength correspond to the maximum growth rate is given by $\omega_{\max }=\frac{2 \pi}{\sqrt{\mu^{(1)}}}$.

Since $\mu_{n}=\frac{n^{2} \pi^{2}}{L^{2}}$, the wave number $n_{\max }$ correspond to the maximum growth rate is given by $n_{\max }=\frac{\sqrt{\mu^{(1)}} L}{\pi}$.

The plot of $n_{\max }$ with respect to $\alpha$ for different values of $\lambda$ are shown in Figure 8. It can be observed that for fixed $\lambda$ and $d$ the value of $n_{\max }$ increases when $\alpha$ increases from $\alpha_{\min }$ to $\alpha_{\max }$. Furthermore, when both $\lambda$ and $\alpha$ increase, the possible minimum and maximum value of $n_{\max }$ as well as the range of $n_{\max }$ increase.

\section{Branching structures}

Now we consider the behaviour of the solutions of the system (1) in one dimensional space when parameters lie in the Turing space. It can be concluded from the system (13) that for large $t$, values of $u$ and $v$ are dominated by (almost depend on) a maximum value of $\operatorname{Re}(\sigma)$ and approach to a spatial variant time invariant patterns. These are wave-like patterns with wave length $\omega_{\max }$ since $\operatorname{Re}(\sigma)$ is maximised at this wave length. Consider the spatial temporal patterns formed by the solutions when parameters lie near the Hopf bifurcation curve $\alpha=\frac{\lambda^{2}}{\sqrt{\lambda-1}}$. For an example consider the case $\lambda=3.2$, $\alpha=6.48596, d=0.001$ and $\Omega=(0,8)$. In this case $L=8, \mu^{(1)}=88.8256$ and the maximum wave number and maximum wave length are $n_{\max }=\frac{\sqrt{\mu^{(1)}} L}{\pi}=12$ and $\omega_{\max }=\frac{2 \pi}{\sqrt{\mu^{(1)}}}=0.66667$, respectively. That is, in this case, it can most probably be expected six (6) branches of the density plot of time evolution of $v$ if a sufficiently large time interval is chosen. The system (4) with the above parameters is solved numerically in one dimensional space using MATLAB built in function pdepe assigning $\Delta x=0.04, \Delta t=0.005$, $\omega=\left(\frac{L}{2}-3 \Delta x, \frac{L}{2}+3 \Delta x\right)$. Error control options are kept in their default values, AbsTol $=10^{-6}$ and 


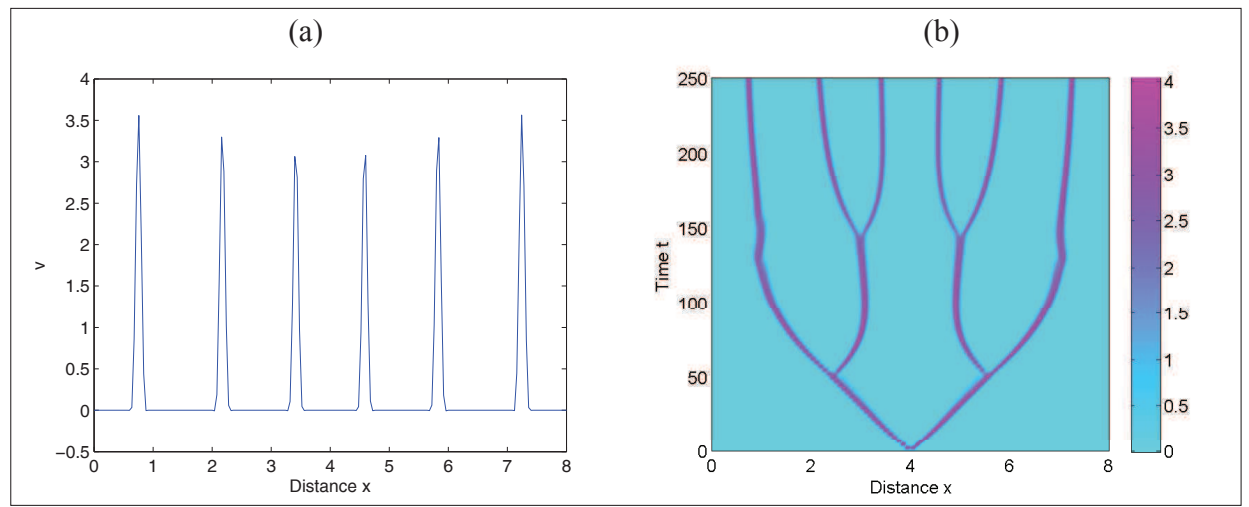

Figure 9: Time invariant spatial patterns and the density plot of time evolution of $v$. (a) Time invariant solution of $v(v$ at $t=250)$; (b) Branching structure formed by time evolution of $v$
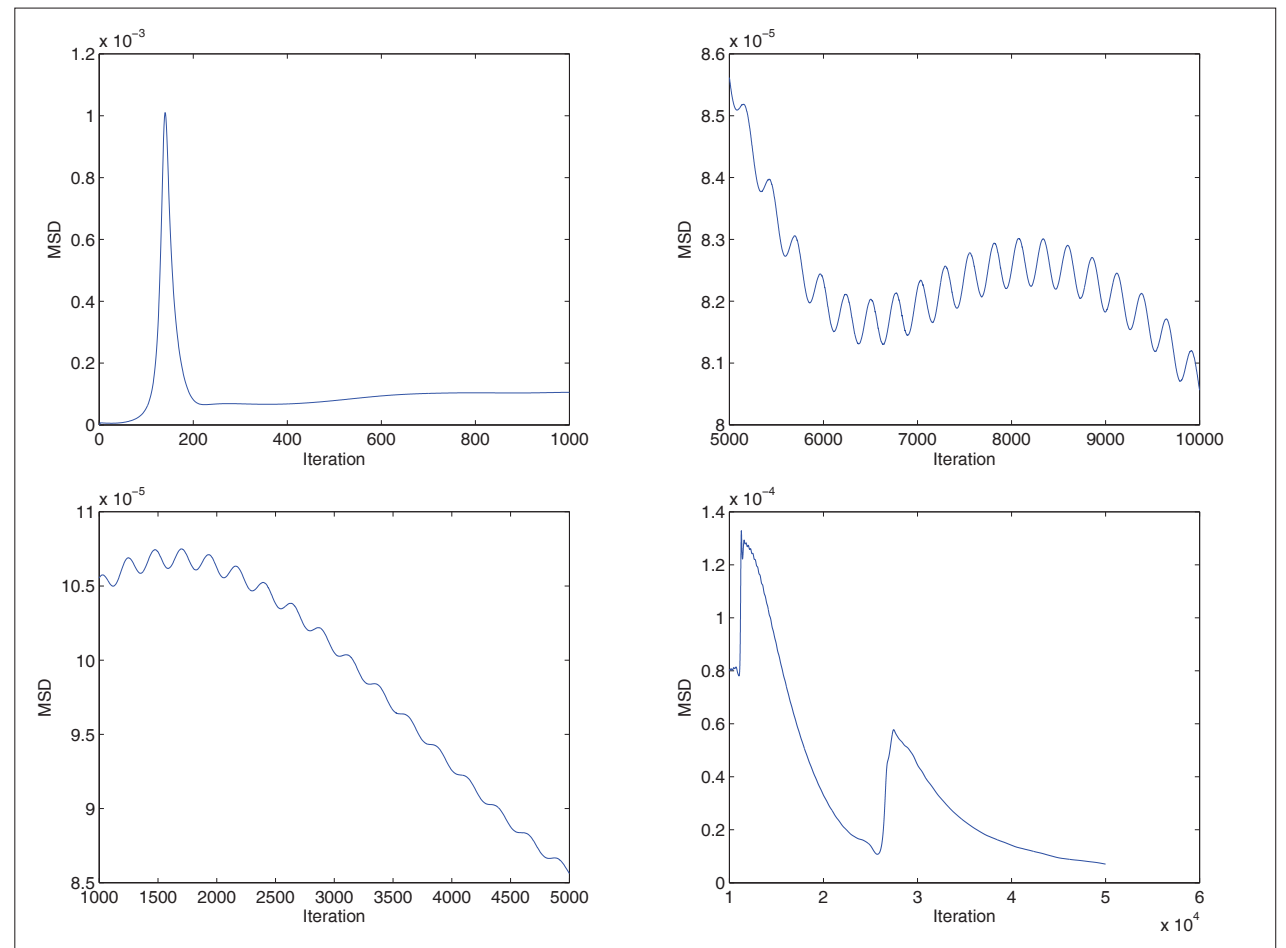

Figure 10: Propagation of mean square difference of the numerical solutions of $v$ between two consecutive iterations

RelTol $=10^{-3}$, respectively. The numerical solutions of $v$ component at large time and the time evolution of $v$ is shown in Figure 9. The default value of RelTol guarantees that $0.1 \%$ accuracy of the solutions in each iteration. The default value of AbsTol guarantees that if zero solutions exist, then in each iteration the absolute value of the deference between the new solution and the previous solution is less than $10^{-6}$. Density plot of the numerical solution of $v$ component shows six branches.
This confirms that the theoretically obtained wave number of the solution at large time is matched with the wave number of the numerical solutions of $v$ at large time.

Suppose that, in the numerical simulation process, space is discretised into $n$ number of sub intervals. Define the mean square difference (MSD) of $v$ between $(i+1)^{\text {th }}$ time step and $i^{\text {th }}$ as follows. 


$$
M S D_{i}=\frac{\sum_{j=1}^{n}\left(v_{j, i+1}-v_{j, i}\right)^{2}}{n}
$$

The propagation of MSD of $v$ is shown in Figure 10 (for clear visibility, the whole range of iterations divided into four sub-ranges and plotted MSD on those sub ranges). It can be observed that MSD is maximised around $140^{\text {th }}$ time step. This maximum value is very small and it is approximately $10^{-3}$. Therefore, numerical values of $v$ are smoothly varying in time. Also as time passed MSD reaches zero. That means the numerical solution of $v$ tends to be at a time invariant state as time passed. Therefore, the convergence and the stability of this numerical process holds.

\section{CONCLUSION}

There are three spatially homogeneous steady states, namely, $S_{1}, S_{2}, S_{3}$ for the model. Turing instability conditions are satisfied only at $S_{2}$. The boundaries of Turing space are determined as functions of model parameters.

For given $\lambda$, the possible maximum value for $d_{c}, \max \left(d_{c}\right)$ is given at the possible minimum value of $\alpha=\alpha_{\min }$ (Figure 2). As $\lambda$ increases, $\max \left(d_{c}\right)$ tends to be $d_{c_{1}}=3-2 \sqrt{2}$. This behaviour is shown in Figure 3 .

For given $\lambda$ and $d$ the instability region of the solutions are bounded by $\alpha_{\min }$ and $\alpha_{\max }$ [see equations (15) and (16)]. According to Figure 5 it can be observed that for given $\lambda$ and $d$, the maximum range for $\mu$ is occurred at $\alpha=\alpha_{\min }$. That is, heterogeneity of the solutions $u$ and $v$ maximise at $\alpha=\alpha_{\min }$ for given $\lambda$ and $d$. Also, Figure 5 shows that as $d$ increases the possible ranges of $\alpha$ and $\mu$ decrease. Therefore, for fixed $\lambda$, the heterogeneity of the solutions of system (1) in a particular domain decreases as $d$ increases.

Density plots of the numerical solutions of $v$ component [see Figure 9(b)] on one dimensional space shows some branching patterns when the parameters lie near the Hopf bifurcation curve, $\alpha=\frac{\lambda^{2}}{\sqrt{\lambda-1}}$. These branching structures are some what similar to the branching structures of branching corals. For fixed parameters, the possible maximum number of branches increases as the domain size increases since $n_{\max }=\frac{\sqrt{\mu^{(1)}} L}{\pi}$. For given parameter values, the critical domain size is $L_{c}=\frac{\pi}{\sqrt{\mu^{(2)}}}$. That is spatial patterns can be expected only if $L>L_{c}$.
Consider the case $D_{u}, D_{v}, H, k, k_{1}$ and domain are fixed. In this case $d$ and $\lambda$ are constants and $\alpha$ is proportional to the nutrient supplying rate $u_{s}$. Therefore, $\alpha$ can be controlled by controlling the nutrient supply. According to Figure $8, n_{\max }$ increases as $\alpha$ increases from $\alpha_{\min }$ to $\alpha_{\max }$. In other words, heterogeneity of the spatial patterns can be controlled by controlling the nutrient supply rate. Also, spatial patterns can be expected only if the nutrient supply rate is controlled in such a way that $\alpha \in\left(\alpha_{\min }, \alpha_{\max }\right)$. According to Figure 7 , for fixed $\lambda$, the growth rate decreases as $\alpha$ increases from $\alpha_{\text {min }}$ to $\alpha_{\max }$. Also, as $\lambda$ increases the maximum growth rate increases and the possible range of $\alpha$ also increases.

\section{REFERENCES}

1. Chindapol N., Kaandorp J.A., Cronemberger C., Mass T. \& Genin A. (2013). Modelling growth and form of the scleractinian coral pocillopora verrucosa and the influence of hydrodynamics. PLOS: Computational Biology 9(1): e1002849

DOI: https://doi.org/10.1371/journal.pcbi.1002849

2. Engelhardt R. (1994). Modelling Pattern Formation in Reaction-Diffusion Systems, Technical Report. University of Copenhagen, Denmark.

3. Kaandorp J.A., Lowe C.P., Frenkel D. \& Sloot P.M.A. (1996). Effect of nutrient diffusion and flow on coral morphology. Physical Review Letters 77(11): 2328 - 2331. DOI: https://doi.org/10.1103/PhysRevLett.77.2328

4. Kaandorp J.A., Sloot P.M.A., Merks R.M.H., Bak R.P.M., Vermeij M.J.A. \& Maier C. (2005). Morphogenesis of the branching reef coral madracis mirabilis. Proceedings of the Royal Society B: Biological Sciences 77: 127133. DOI: https://doi.org/10.1098/rspb.2004.2934

5. Kaandorp J.A., Blom J.G., Verhoef J., Filatov M., Postma M. \& M"uller W.E.G. (2008). Modelling genetic regulation of growth and form in a branching sponge. Proceedings of the Royal Society B: Biological Sciences 275: 2569 - 2575. DOI: https://doi.org/10.1098/rspb.2008.0746

6. Merks R.M.H. (2003a). Branching growth in stony corals: a modelling approach. PhD thesis, Advanced School of Computing and Imaging, University of Amsterdam, The Netherlands.

7. Merks R.M.H. (2003b). Diffusion-limited aggregation in laminar flows. International Journal of Modern Physics $C$ 14(9): $1171-1182$.

DOI: https://doi.org/10.1142/S0129183103005297

8. Merks R.M.H. (2003c). Models of coral growth: spontaneous branching, compactification and laplacian growth assumption. Journal of Theoretical Biology 224(2): $153-166$

DOI: https://doi.org/10.1016/S0022-5193(03)00140-1

9. Merks R.M.H. (2010). Problem solving environment for modelling stony coral morphogenesis. Lecture Notes in Computer Science 2657: 639 - 648

DOI: https://doi.org/10.1007/3-540-44860-8_66 
10. Merks R.M., Hoekstra A., Kaandorp J. \& Sloot P. (2004). Polyp oriented modelling of coral growth. Journal of Theoretical Biology 228: 559576.

DOI: https://doi.org/10.1016/j.jtbi.2004.02.020

11. Mistr S. \& Bercovici D. (2003). A theoretical model of pattern formation in coral reefs. Ecosystems 6: $61-74$. DOI: https://doi.org/10.1007/s10021-002-0199-0

12. Murray J.D. (2003). Mathematical Biology: Spatial Models and Biomedical Applications, volume II. Springer-Verlag, Berlin-Heidelberg, Germany.

13. Somathilake L.W. \& Wedagedera J.R. (2012). On the stability of a mathematical model for coral growth in a tank. British Journal of Mathematics and Computer Science 2(4): 255 - 280.

DOI: https://doi.org/10.9734/BJMCS/2012/1387

14. Somathilake L.W. \& Wedagedera J.R. (2014). A reactiondiffusion type mathematical model for formation of coral patterns. Journal of the National Science Foundation of Sri Lanka 42(4): 341 - 349 .

DOI: https://doi.org/10.4038/jnsfsr.v42i4.7733

15. Turing A. (1952). The chemical basis of morphogenesis. Philosophical Transactions of the Royal Society of London 237(641): $37-72$.

DOI: https://doi.org/10.1098/rstb.1952.0012 